\title{
RANGE INCLUSION FOR CONVEX PROCESSES ON BANACH SPACES; APPLICATIONS IN CONTROLLABILITY
}

\author{
OVIDIU CÂRJĂ
}

\begin{abstract}
We extend the results of Mary Embry [9] on majorisation and range inclusion of bounded linear operators on Banach spaces to the case when one of the two operators is a convex process. An application in controllability is given.
\end{abstract}

\section{INTRODUCTION AND MAIN RESULTS}

The following two theorems concerning range inclusion for bounded linear operators between Banach spaces were proved by Mary Embry [9].

Theorem 1.1. Let $X, Y, Z$ be Banach spaces and let $E \in L(X, Y), F \in$ $L(X, Z)$. Then conditions (i) and (ii) below are equivalent.

(i) $E^{*}\left(Y^{*}\right) \subset F^{*}\left(Z^{*}\right)$;

(ii) $\|E x\| \leq k_{1}\|F x\|, x \in X$, for some $k_{1}>0$.

Here $L(X, Y)$ is the space of all bounded linear operators from $X$ into $Y$, $X^{*}$ is the dual of $X, E^{*}: Y^{*} \rightarrow X^{*}$ is the dual map of $E$.

Theorem 1.2. For $E \in L(Z, Y), F \in L(X, Y)$, we have (iii) $\Rightarrow$ (iv) where

(iii) $E(Z) \subset F(X)$;

(iv) $\left\|E^{*} y^{*}\right\| \leq k_{2}\left\|F^{*} y^{*}\right\|, y^{*} \in Y^{*}$, for some $k_{2}>0$.

Subsequently, these theorems were generalized in different directions: Dolecki and Russell [8] consider $F$ linear, closed with dense domain, Grabiner [11] considers $E$ and $F$ in Theorem 1.2 as bounded multilinear mappings. On the other hand, Pták [16] and Cârjă [6] establish relationships between conditions (i) to (iv) and the following:

(v) $E(S) \subset k_{3} F(S)$ for some $k_{3}>0$;

(vi) $E(S) \subset k_{4} \operatorname{cl} F(S)$ for some $k_{4}>0$.

Throughout the paper, $S$ denotes the unit closed ball of center zero in a normed space, while cl $M$ denotes the closure of the set $M$.

More precisely, we have (iii) $\Leftrightarrow$ (v) and (iv) $\Leftrightarrow$ (vi).

Received by the editors June 5, 1987 and, in revised form, November 3, 1987.

1980 Mathematics Subject Classification (1985 Revision). Primary 52A07, 46A30, 49E15. 
Various applications of these results have been done. See Johnson and Williams [12], Fong [10], Dolecki and Russell [8], Curtain and Pritchard [7], and Cârjă $[4,5,6]$.

Here we extend Theorems 1.1 and 1.2 to the case where $F$ is a convex process and give applications in controllability.

Let $X, Y$ be linear spaces. A convex process from $X$ to $Y$ is a multifunction $F: X \rightarrow 2^{Y}$ (we simply write $F: X \rightarrow Y$ ) whose graph

$$
\operatorname{Gr} F=\{(x, y) \in X \times Y ; y \in F(x)\}
$$

is a convex cone. In the sequel we shall consider convex processes $F$ for which $\mathrm{Gr} F$ has the vertex at the origin. If $\mathrm{Gr} F$ is closed, we say that the convex process is closed. We also note that throughout this paper all linear spaces are considered over the real field.

The domain of $F$ is defined by

$$
D(F)=\{x \in X ; F(x) \neq \varnothing\} .
$$

For any set $C$ in $X$,

$$
F(C)=\bigcup\{F(x) ; x \in C \cap D(F)\} .
$$

Finally, the kernel of $F$ is $\operatorname{Ker} F=\{x \in X ;(x, 0) \in \mathrm{Gr} F\}$.

The convex processes were introduced by Rockafellar [18] in the finite dimensional setting. Subsequently, they have been studied in general linear spaces in connection with optimization problems. See, e.g., Borwein [2] and the references.

The adjoint process $F^{*}: Y^{*} \rightarrow X^{*}$ is defined by

$$
\operatorname{Gr} F^{*}=\left\{\left(y^{*}, x^{*}\right) \in Y^{*} \times X^{*} ;\left(-x^{*}, y^{*}\right) \in(\mathrm{Gr} F)^{+}\right\},
$$

where $(\mathrm{Gr} F)^{+}$is the positive polar cone of $\mathrm{Gr} F$. Recall that the positive polar cone of a set $C$ in $X$ is the set $C^{+}$in $X^{*}$ defined by $C^{+}=\left\{x^{*} \in\right.$ $\left.X^{*} ;\left(x, x^{*}\right) \geq 0, x \in C\right\}$.

Of course, when $F$ is a linear dense defined operator, its adjoint as a linear operator coincides with its adjoint as a convex process.

The main results of this paper are

Theorem 1.3. Let $X, Y, Z$ be Banach spaces, let $E \in L(X, Y)$ and let $F: X \rightarrow$ $Z$ be a convex process. Let us state the conditions:

(a) $E^{*}\left(Y^{*}\right) \subset F^{*}\left(Z^{*}\right)$;

(b) $\|E x\| \leq k_{5}\|y\|$ for any $(x, y) \in \mathrm{Gr} F$ and for some $k_{5}>0$;

(c) $E^{*}(S) \subset k_{6} F^{*}(S)$ for some $k_{6}>0$.

Then $(\mathrm{a}) \Leftrightarrow(\mathrm{b})$ and $(\mathrm{b}) \Leftrightarrow(\mathrm{c})$ with $k_{5}=k_{6}$.

Theorem 1.4. Let $X, Y, Z$ be Banach spaces, let $E \in L(Z, Y)$ and let $F$ : $X \rightarrow Y$ be a closed convex process. Let us state the following conditions:

$$
\left(\mathrm{a}^{\prime}\right) E(Z) \subset F(X) \text {; }
$$


(b') $\left\|E^{*} y^{*}\right\| \leq k_{7}\left\|x^{*}\right\|$ for any $\left(y^{*}, x^{*}\right) \in \mathrm{Gr} F^{*}$ and for some $k_{7}>0$;

(c' $E(S) \subset k_{8} F(S)$ for some $k_{8}>0$;

(d') $E(S) \subset k_{9} \operatorname{cl} F(S)$ for some $k_{9}>0$.

Then $\left(\mathrm{a}^{\prime}\right) \Leftrightarrow\left(\mathrm{c}^{\prime}\right)$ and $\left(\mathrm{b}^{\prime}\right) \Leftrightarrow\left(\mathrm{d}^{\prime}\right)$ with $k_{7}=k_{9}$. If $Z=Y$ and $E=I$ (the identity operator $)$ then $\left(\mathrm{c}^{\prime}\right) \Leftrightarrow\left(\mathrm{d}^{\prime}\right)$.

We first state and prove two lemmas and then prove Theorems 1.3 and 1.4.

We denote by $I_{A}$ the indicator function of the set $A$, i.e.,

$$
I_{A}(x)= \begin{cases}0 & \text { if } x \in A \\ +\infty & \text { otherwise. }\end{cases}
$$

If $A$ is a subset of a linear normed space $X, I_{A}^{*}$ denotes the conjugate of $I_{A}$ which is in fact the support function of $A$. More precisely, we have

$$
I_{A}^{*}\left(x^{*}\right)=\sup \left\{\left(x, x^{*}\right) ; x \in A\right\}, \quad x^{*} \in X^{*} .
$$

If $A$ is a subset of $X^{*}$, then

$$
I_{A}^{*}(x)=\sup \left\{\left(x, x^{*}\right) ; x^{*} \in A\right\}, \quad x \in X .
$$

Lemma 1.1. Let $F: X \rightarrow Y$ be a closed convex process, $X, Y$ being Banach spaces. Then

$$
I_{F(S)}^{*}\left(y^{*}\right)=\left\{\begin{array}{l}
\min \left\{\left\|x^{*}\right\| ;\left(-y^{*}, x^{*}\right) \in \mathrm{Gr} F^{*}\right\}, \\
+\infty \text { if }\left\{x^{*} ;\left(-y^{*}, x^{*}\right) \in \mathrm{Gr} F^{*}\right\} \text { is empty },
\end{array}\right.
$$

and

$$
I_{F^{*}(S)}^{*}(x)=\left\{\begin{array}{l}
\min \{\|y\| ;(y,-x) \in \mathrm{Gr} F\} \\
+\infty \text { if }\{y ;(y,-x) \in \mathrm{Gr} F\} \text { is empty. }
\end{array}\right.
$$

Proof. By definition of the conjugate function we have

$$
I_{F(S)}^{*}\left(y^{*}\right)=I_{(\mathrm{Gr} F) \cap(S \times Y)}^{*}\left(0, y^{*}\right)=\left(I_{\mathrm{Gr} F}+I_{S \times Y}\right)^{*}\left(0, y^{*}\right) .
$$

We now use [19, Theorem 20] to write

$$
\begin{aligned}
& \left(I_{\mathrm{Gr} F}+I_{S \times Y}\right)^{*}\left(0, y^{*}\right) \\
& \quad=\min \left\{I_{\mathrm{Gr} F}^{*}\left(u^{*}, v^{*}\right)+I_{S \times Y}^{*}\left(-u^{*}, y^{*}-v^{*}\right) ; u^{*} \in X^{*}, v^{*} \in Y^{*}\right\} .
\end{aligned}
$$

But

and

$$
I_{A \times Y}^{*}(p, q)=\left\{\begin{array}{cc}
+\infty & \text { if } q \neq 0 \\
I_{A}^{*}(p) & \text { if } q=0
\end{array}\right.
$$

$$
I_{\mathrm{Gr} F}^{*}\left(u^{*}, v^{*}\right)= \begin{cases}0 & \text { if }\left(-u^{*},-v^{*}\right) \in(\mathrm{Gr} F)^{+}, \\ +\infty & \text { in rest. }\end{cases}
$$

Combining all these relations we get (1.1) as claimed.

The proof of (1.2) is similar to that of (1.1). Note that the well-known theorem of bipolars is used in order to obtain that $(\mathrm{Gr} F)^{++}=\mathrm{Gr} F$.

On the other hand, the following lemma is well known and easily verified with a separation theorem. 
Lemma 1.2. If $A, B$ are convex sets in a Banach space $X$ then $A \subset \operatorname{cl} B$ if and only if $I_{A}^{*}\left(x^{*}\right) \leq I_{B}^{*}\left(x^{*}\right), x^{*} \in X^{*}$. If $A, B$ are convex sets in $X^{*}$ then $A \subset \mathrm{cl} B$ if and only if $I_{A}^{*}(x) \leq I_{B}^{*}(x), x \in X$.

Proof of Theorems 1.3 and 1.4. The equivalence $\left(\mathrm{a}^{\prime}\right) \Leftrightarrow\left(\mathrm{c}^{\prime}\right)$ (hence $(\mathrm{a}) \Leftrightarrow(\mathrm{c})$ ) follows easily by the open mapping theorem for convex processes [17]. To obtain the desired result we only have to consider in the quoted theorem the closed convex process $E^{-1} F$. Now we take in Lemma $1.2 A=E(S), B=k_{9} F(S)$ and deduce that $\left(\mathrm{d}^{\prime}\right)$ is equivalent to: $\left\|E^{*} y^{*}\right\| \leq k_{9} I_{F(S)}^{*}\left(y^{*}\right), y^{*} \in Y^{*}$. By (1.1) it follows that $\left(\mathrm{b}^{\prime}\right) \Leftrightarrow\left(\mathrm{d}^{\prime}\right)$. To prove $(\mathrm{b}) \Leftrightarrow(\mathrm{c})$, we apply Lemma 1.2 with $A=E^{*}(S), B=k_{6} F^{*}(S)$ and then use (1.2) and the fact that $F^{*}(S)$ is closed.

We conclude the proof by showing that if $Z=Y$ and $E=I$ then $\left(\mathrm{c}^{\prime}\right) \Leftrightarrow$ $\left(\mathrm{d}^{\prime}\right)$. Indeed, it is easy to see that $F(S)$ is ideally convex (that is, for every $\left(\lambda_{n}\right)_{n \geq 1}, \lambda_{n} \geq 0, \sum_{n \geq 1} \lambda_{n}=1$ and for every bounded sequence $\left(y_{n}\right)_{n \geq 1} \subset$ $F(S)$ we have $\left.\sum_{n \geq 1} \lambda_{n} y_{n} \in F(S)\right)$. By a result of Lifsic [13] we deduce that $F(S)$ and $\operatorname{cl} F(S)$ have the same interior. Therefore $\left(\mathrm{c}^{\prime}\right) \Leftrightarrow\left(\mathrm{d}^{\prime}\right)$ and the proof is complete.

Remark that if $E$ is not the identity operator, the implication $\left(\mathrm{d}^{\prime}\right) \Rightarrow\left(\mathrm{c}^{\prime}\right)$ is generally not true even if $F$ is a linear operator. See [1]. Finally, we remark that in the special case $E=I$ Theorems 1.3 and 1.4 are closely related to Theorem 2.1 in [3].

\section{APPLICATIONS IN CONTROLLABILITY THEORY}

In this section we shall apply the theory developed in the preceding section to the study of controllability of linear systems when the constraint set of controls is a convex cone.

Let $X$ be a reflexive Banach space and $S(t), t \geq 0$, be a linear semigroup of class $c_{0}$ in $X$. Let $A$ be the infinitesimal generator of $S(t)$. See, e.g., [14] for the necessary background for linear semigroups in Banach spaces.

Consider the control system described by the equation

$$
x^{\prime}(t)=A x(t)+B u(t), \quad t \geq 0,
$$

where $B$ is a bounded linear operator from a reflexive Banach space $U$ into $X$. The solution of equation (2.1) is understood in the mild sense, i.e.,

$$
x(t)=S(t) x_{0}+\int_{0}^{t} S(t-s) B u(s) d s, \quad t \geq 0 .
$$

Let $\Omega$ be a closed convex cone with vertex at zero, $\Omega \subset U$. Let us fix $T>0$ and consider the constraint set of controls as

$$
U_{\mathrm{ad}}^{p}=\left\{u \in L^{p}(0, T ; U) ; u(t) \in \Omega \text { a.e. }\right\}, \quad 1 \leq p \leq \infty .
$$

We say that the control system $(2.1)$ is null controllable at time $T$ in the constraint set $U_{\mathrm{ad}}^{p}$ if for each $x_{0} \in X$ there exists $u \in U_{\mathrm{ad}}^{p}$ such that $x(0)=x_{0}$ and $x(T)=0$, where $x(\cdot)$ is given by $(2.2)$. 
Theorem 2.1. The control system (2.1) is null controllable at time $T$ in the constraint set $U_{\mathrm{ad}}^{p}, 1<p \leq \infty$, if and only if there exists $k>0$ such that

$$
\left(\int_{0}^{T}\left\|B^{*} S^{*}(s) x^{*}+w(s)\right\|^{q} d s\right)^{1 / q} \geq k\left\|S^{*}(T) x^{*}\right\|,
$$

for every $w \in L^{q}\left(0, T ; U^{*}\right), w(s) \in \Omega^{+}$a.e. and for every $x^{*} \in X^{*}$, where $1 / p+1 / q=1$. More precisely, all points from the closed ball of center zero and radius $k$ can be transferred to zero at time $T$ by controls $u \in U_{\mathrm{ad}}^{p}$ with $\|u\|_{L^{p}} \leq 1$ if and only if (2.4) holds.

Before proving this theorem, we examine an example of convex process and its associated adjoint. See [2] for details.

Example 2.1. Let $V: X \rightarrow Y$ be densely defined linear. Let $K \subset X$ and $S \subset Y$ be convex cones. Set

$$
H(x)= \begin{cases}V x+S, & x \in K, \\ \varnothing, & x \notin K .\end{cases}
$$

It is easily seen that $H: X \rightarrow Y$ is a convex process. The adjoint $H^{*}$ is given by

$$
H^{*}\left(y^{*}\right)= \begin{cases}V^{*} y^{*}-K^{+}, & y^{*} \in S^{+}, \\ \varnothing, & y^{*} \notin S^{+} .\end{cases}
$$

Proof of Theorem 2.1. Consider the convex process

$$
\begin{gathered}
F: X^{*} \rightarrow L^{q}\left(0, T ; U^{*}\right), \\
F X^{*}=B^{*} S^{*}(T-\cdot) x^{*}+\widetilde{\Omega}
\end{gathered}
$$

where $\widetilde{\Omega}=\left\{\tilde{u} \in L^{q}\left(0, T ; U^{*}\right) ; \tilde{u}(s) \in \Omega^{+}\right.$a.e. $\}$.

This is a particular case of Example 2.1.

It is easily shown that the adjoint of the operator $V: X^{*} \rightarrow L^{q}\left(0, T ; U^{*}\right)$, $V x^{*}=B^{*} S^{*}(T-\cdot) x^{*}$ is given by

$$
\begin{gathered}
V^{*}: L^{p}(0, T ; U) \rightarrow X, \\
V^{*} u=\int_{0}^{T} S(T-s) B u(s) d s .
\end{gathered}
$$

On the other hand, $\widetilde{\Omega}^{+}=U_{\text {ad }}^{p}$. So the adjoint of $F$ (via Example 2.1) is

$$
\begin{gathered}
F^{*}: L^{p}(0, T ; U) \rightarrow X, \\
F^{*} u= \begin{cases}\int_{0}^{T} S(T-s) B u(s) d s & \text { if } u \in U_{\mathrm{ad}}^{p}, \\
\varnothing & \text { otherwise. }\end{cases}
\end{gathered}
$$

Observe now that the control system (2.1) is null controllable at time $T$ in the constraint set $U_{\mathrm{ad}}^{p}$ if and only if $-S(T)(X) \subset F^{*}\left(L^{p}(0, T ; U)\right)$. 
Applying Theorem 1.3 with $E=-S^{*}(T)$ and with $F$ defined above we end the proof of Theorem 2.1.

Remark that if $\Omega=U,(2.4)$ becomes

$$
\left(\int_{0}^{T}\left\|B^{*} S^{*}(s) x^{*}\right\|^{q} d s\right)^{1 / q} \geq k\left\|S^{*}(T) x^{*}\right\|, \quad x^{*} \in X^{*},
$$

which is the well-known condition of continuous observability of the adjoint system $[8,6,7]$.

We conclude with some remarks on approximate controllability.

We say that the control system (2.1) is approximately null controllable at time $T$ in the constraint set $U_{\text {ad }}^{p}$ if for every $\varepsilon>0$ and every $x_{0} \in X$ there exists $u \in U_{\text {ad }}^{p}$ such that $x(0)=x_{0}$ and $\|x(T)\|<\varepsilon$, where $x(\cdot)$ is given by (2.2).

Let us define the reachable set from $x_{0}$ by

$$
A^{p}\left(T ; x_{0}\right)=\left\{x(T) ; x(\cdot) \text { is given by }(2.2), x(0)=x_{0}, u \in U_{\mathrm{ad}}^{p}\right\} .
$$

Theorem 2.2. $0 \in \operatorname{cl} A^{p}\left(T ; x_{0}\right), 1 \leq p<\infty$, if and only if $B^{*} S^{*}(s) x^{*} \in \Omega^{+}$on $[0, T]$ implies $\left(x_{0}, S^{*}(T) x^{*}\right) \leq 0$.

Proof. Consider the closed convex process $F: L^{p}(0, T ; U) \rightarrow X$ given by

$$
F(u)= \begin{cases}\int_{0}^{T} S(T-s) B u(s) d s & \text { if } u \in U_{\mathrm{ad}}^{p} \\ \varnothing & \text { otherwise. }\end{cases}
$$

This is also a particular case of Example 2.1.

Taking into account that $\left(U_{\text {ad }}^{p}\right)^{+}=\widetilde{\Omega}$, the adjoint of $F$ (via Example 2.1) is $F^{*}: X^{*} \rightarrow L^{q}\left(0, T ; U^{*}\right)$,

$$
F^{*} x^{*}=B^{*} S^{*}(T-\cdot) x^{*}-\widetilde{\Omega}, \quad x^{*} \in X^{*} .
$$

Observe that $0 \in \operatorname{cl} A^{p}\left(T ; x_{0}\right)$ if and only if $-S(T) x_{0} \in \operatorname{cl} F\left(L^{p}(0, T ; U)\right)$.

We apply now [2, Corollary 6.3] (see also [20]) which states that for a closed convex process $F: X \rightarrow Y, X, Y$ being locally convex spaces, we have $\operatorname{cl} F(X)=\left(\operatorname{Ker} F^{*}\right)^{+}$.

Hence $0 \in \operatorname{cl} A^{p}\left(T ; x_{0}\right)$ if and only if $-S(T) x_{0} \in\left(\operatorname{Ker} F^{*}\right)^{+}$. On the other hand, by $(2.5),-S(T) x_{0} \in\left(\operatorname{Ker} F^{*}\right)^{+}$if and only if $B^{*} S^{*}(T-s) x^{*} \in \Omega^{+}$a.e. $s \in[0, T]$ implies $\left(S(T) x_{0}, x^{*}\right) \leq 0$. Along with the continuity of $S^{*}(\cdot) x^{*}$ the latter proves our theorem.

Corollary 2.1. The control system (2.1) is approximately null controllable at time $T$ in the constraint set $U_{\mathrm{ad}}^{p}, 1 \leq p<\infty$, if and only if $B^{*} S^{*}(s) x^{*} \in \Omega^{+}$ on $[0, T]$ implies $S^{*}(T) x^{*}=0$.

Proof. This is a direct consequence of Theorem 2.2. Indeed, the control system (2.1) is approximately null controllable at time $T$ in the constraint set $U_{\text {ad }}^{p}$ if and only if $0 \in \operatorname{cl} A^{p}\left(T ; x_{0}\right)$ for every $x_{0} \in X$.

For $1<p<\infty$, Theorem 2.2 is proved directly in [15]. Other applications in controllability of the results of $\S 1$ will be given elsewhere. 


\section{REFERENCES}

1. R. Bouldin, A counterexample in the factorization of Banach space operators, Proc. Amer. Math. Soc. 68 (1978), 327.

2. J. M. Borwein, Adjoint process duality, Math. Oper. Res. 8 (1983), 403-434.

3. __ Stability and regular points of inequality systems, J. Optim. Theory Appl. 48 (1986), 53-64.

4. O. Cârjă, On continuity of the minimal time function for distributed control systems, Boll. Un. Mat. Ital. B (6) 3 (1984), 563-581.

5. __ A note on admissible null controllability and on variational perturbations of the minimum time problem, An. Ştiinţ. Univ. “Al. I. Cuza” Iaşi Sect. I a Mat. 32 (1986), 13-19.

6. $\ldots$, On constraint controllability of linear systems in Banach spaces, J. Optim. Theory Appl. 56 (1988),215-225.

7. R. F. Curtain and A. J. Pritchard, Infinite dimensional linear systems theory, Springer-Verlag, Berlin, 1978.

8. S. Dolecki and D. L. Russell, A general theory of observation and control, SIAM J. Control Optim. 15 (1977), 185-220.

9. M. R. Embry, Factorizations of operators on Banach spaces, Proc. Amer. Math. Soc. 38 (1973), 587-590.

10. C. K. Fong, Range inclusion for normal derivation, Glasgow J. Math. 25 (1984), 255-262.

11. S. Grabiner, Operator ranges and invariant subspaces, Indiana Univ. Math. J. 28 (1979), 845857.

12. B. E. Johnson and J. P. Williams, The range of a normal derivation, Pacific J. Math. 58 (1975), 105-122.

13. E. A. Lifsic, Ideally convex sets, Funkcional Anal. i Priložen 4 (1970), 76-77. (Russian)

14. A. Pazy, Semigroups of linear operators and applications to partial differential equations, Springer-Verlag, New York, 1983.

15. G. Peichl and W. Schappacher, Constrained controllability in Banach spaces, SIAM J. Control Optim. 24 (1986), 1261-1275.

16. V. Pták, Some metric aspects of the open mapping and closed graph theorems, Math. Ann. 163 (1966), 95-104.

17. S. Robinson, Normed convex processes, Trans. Amer. Math. Soc. 174 (1972), 127-140.

18. R. T. Rockafellar, Monotone processes of convex and concave type, Mem. Amer. Math. Soc. No. $77,1967$.

19. __ Conjugate duality and optimization, SIAM, Philadelphia, Pa., 1974.

20. C. Zălinescu, Letter to the editor on Borwein's paper "Adjoint process duality", Math. Oper. Res. 11 (1986), 692-698.

Department of MATHEMatics, University of IASI, IASI 6600, Romania 